June 21, 2021

WIS-94/27/Jul-PH

hep-ph/9407324

\title{
Naturally Light Leptoquarks
}

\author{
Ernest Baver and Miriam Leurer* \\ Department of Particle Physics \\ The Weizmann Institute \\ Rehovot 76100 \\ ISRAEL
}

\begin{abstract}
Light first generation leptoquarks are being hunted for in HERA and at FNAL and there are various proposals for further searches in future machines. Such leptoquarks are however problematic from a theoretical point of view: Low energy precision measurements imply strong constraints on the couplings of the leptoquarks, and up till now the fulfilment of these constraints seemed extremely unnatural. Here we show that horizontal symmetries, which are very conventional and widely used in the literature for completely different purposes, can suppress the unwanted couplings. Therefore light first generation leptoquarks can be natural.
\end{abstract}

\footnotetext{
*Address after October 1: Elta, P.O. Box 330, Ashdod, Israel
} 


\section{Introduction}

There has been an increasing interest in light first generation leptoquarks in recent years, due to the exciting possibility of observing such particles in the electron-proton machine HERA [1]. The search for low-lying leptoquarks is supported theoretically by many beyond-standard models which predict their existence [2]. However, close phenomenological studies show that leptoquarks are troublesome [3-9]: They can induce proton decay and various FCNC processes, and they can enhance leptonic decays of pseudoscalar mesons by many orders of magnitude. Up to now, these potential problems with leptoquarks have been circumvented simply by demanding that the leptoquarks couplings obey a list of strong constraints. Here we point out symmetries which naturally lead to the fulfilment of all the desired constraints. The symmetries we propose are not invented for the sake of leptoquarks but are rather conventional symmetries which have been used previously in the literature for other purposes.

The paper is organized as follows: In the next section we describe in some detail the phenomenological troubles that leptoquarks may lead to and the consequent list of constraints on their couplings. We also discuss the general properties of the symmetries that we propose for the suppression of the unwanted couplings. Section 3 contains a specific model that incorporates such symmetries. Section 4 lists the leptoquarks couplings in the model and section 5 discusses the success of the model in evading the phenomenological constraints. The last section is a short summary and overview.

\section{The unwanted leptoquark couplings}

The processes that lead to the strongest bounds on the leptoquark mass $(M)$ and couplings $(g)$ are:

(i) Proton decay [4] which is induced when the leptoquark has also diquark couplings. The proton decay bound, $M / g \gtrsim 10^{16} \mathrm{GeV}$, can be avoided by requiring that the diquark couplings vanish.

(ii) Flavour changing neutral current (FCNC) processes, which are induced when the leptoquark couples to a few generations in either the lepton or the quark sector. The strongest bound arises from $K_{L} \longrightarrow \mu e[3,9]$ and is typically $M / g \gtrsim 100 \mathrm{TeV}$. The FCNC bounds are circumvented by demanding that the leptoquarks couple "diagonally", namely, they couple to a single generation in the lepton sector and to a single generation in the quark sector. The diagonality requirement cannot always be fully satisfied $[7,8]$ : In the 
case of leptoquarks that couple to left-handed quarks, the CKM rotation always induces some nondiagonality in the quark sector, leading to an unavoidable FCNC bound $M / g^{2} \gtrsim$ few $\mathrm{TeV}$.

(iii) Enhancement of the leptonic decays of the pion and other pseudoscalars. The bound is particularly strong for leptoquarks that couple to both left-handed (LH) and right-handed (RH) quarks $[5,6,8]: M / g \gtrsim 100 \mathrm{TeV}$. It is avoided by demanding that the leptoquarks couple chirally, namely that they couple either to LH or to RH quarks but not to both. Even when the chirality requirement is satisfied, there are some leptoquarks (those that couple to LH quarks and to LH leptons) which still contribute significantly to leptonic $\pi$ decay $[6,8]$. This contribution leads to an unavoidable bound: $M / g \gtrsim$ few TeV.

(iv) Atomic parity violation: Any first generation leptoquark induces a significant and unavoidable new contribution to atomic parity violation [8], and this leads to bounds which typically read: $M / g \gtrsim$ few hundred $\mathrm{GeV}$.

Summarizing the above list, there are some bounds on leptoquarks that are unavoidable and cannot be circumvented, all of them at the $\mathrm{TeV}$ scale. But the most severe bounds, which send the leptoquark scale to $100 \mathrm{TeV}$ or even to $10^{16} \mathrm{GeV}$ can be avoided if the leptoquarks obey the following constraints: They do not couple to diquarks and they couple diagonally and chirally.

What symmetries could suppress all the unwanted couplings? Clearly, the diquark couplings can be avoided by the conservation of baryon or lepton number or some combination of these. It is also easy to protect the nondiagonal couplings in the lepton sector by imposing separate conservation of the three lepton numbers: electron, muon and tau. The message of our work is that the nondiagonal couplings in the quark sector as well as the nonchiral couplings can be suppressed by the horizontal symmetries which are widely discussed in the literature as an explanation for the pattern and hierarchy in the fermion mass matrices.

The horizontal symmetries have two important characteristics: (i) They, by definition, distinguish the generations, and therefore are likely to favour the leptoquark coupling to a particular generation and suppress its coupling to other generations. This may force the leptoquark couplings to be "diagonal". (ii) They typically also distinguish the LH and RH components of each fermion, namely, the two components carry different horizontal quantum numbers. Therefore such symmetries favour the coupling of a particular chiral component of a given quark and suppress the coupling to the other chirality, so that the leptoquark couplings are forced to be "chiral". 
In the next section we present a leptoquark model which is supplemented by a horizontal symmetry that suppresses the unwanted couplings.

\section{A model}

There are five possible scalar leptoquark multiplets, and we chose to concentrate on the multiplet that is motivated by $E_{6}$ superstring models. This leptoquark is a singlet of $S U(2)_{W}$ and carries $\frac{1}{3}$ unit of electromagnetic charge.

As for our choice of the horizontal symmetry: in addition to its traditional task of "explaining" the pattern and the hierarchy in the fermion mass matrices, the symmetry should also provide the mechanism for suppressing unwanted leptoquark couplings. In a recent series of papers [10-12] it was shown that abelian horizontal symmetries combined with supersymmetry provide a particularly efficient mechanism for suppressing unwanted couplings. Furthermore, since we worry about FCNC processes which may be induced by the leptoquark, we turn to a class of models which were especially constructed to suppress FCNC, the "quark-squark-alignment" (QSA) models [11].

The model we present here has the following symmetries: The standard $S U(3)_{C} \times$ $S U(2)_{W} \times U(1)_{Y}$ gauge symmetry, supersymmetry, baryon number, and a horizontal symmetry which commutes with SUSY and includes the three leptonic quantum numbers (electron, muon and tau) and a $Z_{8} \times Z_{7} \subset U(1)_{H_{1}} \times U(1)_{H_{2}}$.

The spectrum includes three families of quarks and leptons, two Higgs multiplets and two leptoquark multiplets*. Table 1 lists the chiral multiplets of our model and their gauge representations and table 2 lists their horizontal charges $\left(H_{1}, H_{2}\right)$.

Supersymmetry as well as the horizontal symmetry must be broken at low energies. We assume that SUSY is softly broken. As for the horizontal symmetry we follow $[13,10-$ 12 ] and assume that it is explicitly broken in a perturbative manner namely, terms that break the horizontal symmetry are allowed but suppressed according to the following rule: For any term in the Lagrangian that is carrying nontrivial horizontal charges, let $m_{i}(i=1,2)$ be its $i$ 'th horizontal charge modulo $N_{i}^{\dagger}$. The term is then suppressed by

*If we had had only one leptoquark multiplet, then the sleptoquark would have made the gauge symmetry anomalous.

†Since the horizontal symmetries of the Lagrangian are the discrete $Z_{N_{i}}$ rather than the full $U(1)_{H_{i}}$, the symmetry breaking is quantified by the $m_{i}$ 's. 


\begin{tabular}{|l|l|c|}
\hline Supermultiplet & particle content & gauge representation \\
\hline$Q_{i}$ & $\left(\tilde{q}_{i}, q_{i}\right)$ & $(3,2)_{+\frac{1}{6}}$ \\
\hline$L_{i}$ & $\left(\tilde{l}_{i}, l_{i}\right)$ & $(1,2)_{-\frac{1}{2}}$ \\
\hline$U_{i}^{c}$ & $\left(\tilde{u}_{i}^{c}, u_{i}^{c}\right)$ & $(\overline{3}, 1)_{-\frac{2}{3}}$ \\
\hline$D_{i}^{c}$ & $\left(\tilde{d}_{i}^{c}, d_{i}^{c}\right)$ & $(\overline{3}, 1)_{+\frac{1}{3}}$ \\
\hline$E_{i}^{c}$ & $\left(\tilde{e}_{i}^{c}, e_{i}^{c}\right)$ & $(1,1)_{+1}$ \\
\hline$\Phi_{u}$ & $\left(\phi_{u}, \tilde{\phi}_{u}\right)$ & $(1,2)_{+\frac{1}{2}}$ \\
\hline$\Phi_{d}$ & $\left(\phi_{d}, \tilde{\phi}_{d}\right)$ & $(1,2)_{-\frac{1}{2}}$ \\
\hline$S$ & $(s, \tilde{s})$ & $(\overline{3}, 1)_{+\frac{1}{3}}$ \\
\hline$S^{\prime}$ & $\left(s^{\prime}, \tilde{s}^{\prime}\right)$ & $(3,1)_{-\frac{1}{3}}$ \\
\hline
\end{tabular}

Table 1: The chiral spectrum of the model. Supersymmetric multiplets (in the first column) are denoted by capital letters. "Particles" and "sparticles" (in the second column) are denoted by the corresponding small letters, and the sparticles are further provided with a tilde. Here particles are the standard model fermions, the Higgs scalars and the leptoquark. The subscript $i=1,2,3$ is a generation index.

$$
\begin{array}{lll}
Q_{1}(3,0) & Q_{2}(0,1) & Q_{3}(0,0) \\
L_{1}(1,1) & L_{2}(3,0) & L_{3}(3,3) \\
U_{1}^{c}(-2,3) & U_{2}^{c}(1,0) & U_{3}^{c}(0,0) \\
D_{1}^{c}(-1,2) & D_{2}^{c}(4,-1) & U_{3}^{c}(0,1) \\
E_{1}^{c}(1,2) & E_{2}^{c}(2,0) & E_{3}^{c}(0,4) \\
\Phi_{u}(0,0) & \Phi_{d}(0,0) & \\
S(4,-1) & S^{\prime}(-4,-6) &
\end{array}
$$

Table 2: The $\left(H_{1}, H_{2}\right)$ quantum numbers of the matter multiplets in the model. The $S$ and $S^{\prime}$ leptoquark multiplets carry $(-1)$ and $(+1)$ units of electronic lepton number and no muon or tau number. The horizontal symmetry commutes with SUSY (it is not an $R$ symmetry). Note that $U(1)_{\mathrm{H}_{1}} \times U(1)_{\mathrm{H}_{2}}$ is free of $Q C D$ anomalies. 
$\epsilon_{1}^{m_{1}} \epsilon_{2}^{m_{2}}$. The $\epsilon_{i}$ 's in our model are:

$$
\epsilon_{1}=\lambda, \quad \epsilon_{2}=\lambda^{2}
$$

where $\lambda=0.2 \approx \sin \theta_{c}$.

In the quark sector, the model is almost identical to the QSA model of ref [12]. The main difference is that the up quark horizontal numbers are somewhat different, so that the ratio of the up quark and top quark masses fits better the new results from CDF [14]. Our model has all the good features of the QSA model of [12]. In particular, the alignment of the quark-squark mass matrices ensures that no FCNC troubles arise from the contributions of squark-gluino loop to $K-\bar{K}$ and $D-\bar{D}$ mixing, even if the squarks are not degenerate.

In the lepton sector, our model provides the leptons with masses of the correct order of magnitude. The separate conservation of the three lepton number provides a safe protection against FCNC processes in this sector.

\section{The leptoquark couplings}

In this section we discuss all the renormalizable couplings of the model that involve the leptoquarks, both in the superpotential and in the soft supersymmetry breaking terms. We will also see how SUSY together with the horizontal symmetry suppress the unwanted couplings.

There are two types of terms in the supersymmetric potential that involve the leptoquarks: Bilinear terms, which are particularly important since they provide mass for the sleptoquarks, and trilinear terms which are responsible for the leptoquarks' Yukawa couplings to leptons and quarks.

The bilinear term in the supersymmetric potential is

$$
M S S^{\prime}
$$

where $M$ is a mass parameter (which is not suppressed by the horizontal symmetry). When considering the value of $M$ we must take into account two opposing requirements: On the one hand $M$ should not be too small, since it provides the only contribution to the sleptoquark masses. These should not be too light to avoid conflict with LEP precision measurements [15]. On the other hand, $M$ should not be too large since it contributes to 
the leptoquark masses and we are interested in the case of light leptoquarks. We satisfy both requirements by choosing $M$ to be of the order of a few hundred $\mathrm{GeV}$.

The trilinear terms in the supersymmetric potential are given by:

$$
G_{L i j} L_{i} Q_{j} S+G_{R i j} E_{i}^{c} U_{j}^{c} S^{\prime}
$$

The order of magnitude values of the $G_{L}$ and $G_{R}$ matrices can be deduced from the horizontal symmetries of the model. In the quark interaction basis we find:

$$
G_{L}=g\left(\begin{array}{ccc}
1 & \lambda^{7} & \lambda^{5} \\
0 & 0 & 0 \\
0 & 0 & 0
\end{array}\right) \text { and } G_{R}=g\left(\begin{array}{ccc}
\lambda^{15} & \lambda^{12} & \lambda^{11} \\
0 & 0 & 0 \\
0 & 0 & 0
\end{array}\right)
$$

where $g$ is some typical unsuppressed coupling. The matrices above do not give the exact values of the matrix elements, only their relative order of magnitude in powers of $\lambda$. Note that the last two rows of $G_{L}$ and $G_{R}$ vanish as a consequence of the separate conservation of the three lepton numbers.

The $G_{R}$ couplings are strongly suppressed and can practically be ignored. This is very useful because soft supersymmetry breaking terms can lead to a significant mixture of the $s$ and $s^{\prime *}$ leptoquarks. If $G_{R}$ was not suppressed we would have found ourselves with very non-chiral leptoquarks leading to severe problems in leptonic $\pi$ decays.

The nondiagonal couplings in $G_{L}$ are so suppressed that they too can be ignored. However, $G_{L}$ should still be rotated to the mass basis in both the up and down quark sectors (see [12]) and in this process new nondiagonal couplings arise:

$$
G_{L}^{u}=g\left(\begin{array}{ccc}
1 & \lambda & \lambda^{3} \\
0 & 0 & 0 \\
0 & 0 & 0
\end{array}\right), \quad G_{L}^{d}=g\left(\begin{array}{ccc}
1 & \lambda^{5} & \lambda^{3} \\
0 & 0 & 0 \\
0 & 0 & 0
\end{array}\right)
$$

Note that even after the rotation to the mass basis the nondiagonal terms in the down sector are very suppressed and have no practical significance. This is a result of our using a QSA model, where the rotation between the interaction basis and the down mass basis is particularly small. This is useful because FCNC bounds are especially strict for the down-like quarks.

The only couplings of phenomenological importance in (4.4) are the diagonal $G_{L 11}^{u}$ and $G_{L 11}^{d}$ which contribute to $\pi \longrightarrow e \nu$ decay and atomic parity violation, and the nondiagonal $G_{L 12}^{u}$ which contributes to $D-\bar{D}$ mixing. Identifying $g \equiv G_{L 11}$, we find, up to corrections 
of order $\lambda^{2}$ :

$$
\begin{gathered}
\left|G_{L 11}^{u}\right|=\left|G_{L 11}^{d}\right|=|g| \\
\left|G_{L 12}^{u}\right|=|g| \sin \theta_{C}
\end{gathered}
$$

where $\theta_{C}$ is the Cabibbo angle.

Turning to the supersymmetry breaking terms: There are three point vertices involving a squark, a slepton and a leptoquark. These do not cause any phenomenological problems and will not be further discussed. There are also new contributions to the leptoquarks masses, which we parameterize by:

$$
\left(m^{2} s s^{\prime}+\text { h.c. }\right)+m_{1}^{2} s s^{*}+m_{2}^{2} s^{\prime} s^{*}
$$

where all the parameters $m, m_{1}$ and $m_{2}$ are of the order of the SUSY breaking scale (weak scale). The $m$ parameter is responsible for the mixture of $s$ and $s^{\prime *}$ leptoquarks but since the Yukawa couplings of $s^{\prime}$ are very strongly suppressed this mixing is not significant and will be ignored for the sake of simplicity. Altogether the $s^{\prime}$ leptoquark can be ignored from now on: Its direct couplings to quarks and leptons are negligible and we ignore its mixing with $s$.

In closing this section, we mention that the Lagrangian of our model has the standard model gauge symmetry, supersymmetry, the global horizontal symmetry discussed in the previous section (including the three separate lepton numbers and baryon number), and no other continuous accidental symmetry. We therefore do not need to worry about the possibility of (pseudo) Goldstone bosons in the low lying spectrum.

\section{5 "Measuring" the success of the model}

In this section we will "test" our model, namely we will check to what extent the bounds on the leptoquark parameters are evaded. First, we recall that there are TeV scale bounds that cannot be avoided so that the main task of the symmetries in the model was to get rid of the higher scale bounds (Pati-Salam scale, GUT scale). It is easy to see that here our model is indeed successful: The GUT scale bounds are avoided because diquark couplings do not exist. The Pati-Salam scale bounds are also avoided: the bounds from leptonic $\pi$ decays is circumvented because nonchiral couplings are very severely suppressed. The bounds from FCNC processes are also avoided because the couplings in the lepton sector are exactly diagonal, in the down quark sector the deviation from 
diagonality is so small that it can safely be ignored, and in the up quark sector nondiagonal couplings are suppressed by $\sin \theta_{C}$, and consequently the FCNC bounds from this sector are at the $\mathrm{TeV}$ scale (see $[7,8])$.

Now that we are assured that the bounds on the $s$ leptoquark are at the "unavoidable scale", we go into a more detailed test: We will compare the actual values of the bounds on $s$ in our model with the unavoidable bounds that apply to any leptoquark which is in the $(\overline{3}, 1)_{\frac{1}{3}}$ representation of $S U(3)_{C} \times S U(2)_{W} \times U(1)_{Y}$ and couples to LH quarks. The absolutely unavoidable bounds were studied in [8]: One arises from leptonic $\pi$ decays (there is also a weaker bound from atomic parity violation):

$$
M / g \geq 3.4 \mathrm{TeV} \quad \text { at } 95 \% \mathrm{CL} \text {. }
$$

The other bound arises from FCNC processes which are unavoidable for leptoquarks that couple to LH quarks. By a fine-tuned division of the FCNC between the down and up sectors one can minimize the FCNC bound to:

$$
M / g^{2} \geq 2.8 \mathrm{TeV} \quad \text { at } 95 \% \mathrm{CL}
$$

Turning to the corresponding bounds on the $s$ leptoquark in our model we note that the leptonic $\pi$ decay bound on $s$ is identical to (5.1). The FCNC bound on $s$ arises from $D-\bar{D}$ mixing. There are two contributions to this process: One of them arises from a leptoquark-electron box diagram and was already discussed in $[7,8]$. The other contribution arises from a sleptoquark-selectron loop. Adding the two contributions we find:

$$
\Delta M_{D}=\frac{1}{192 \pi^{2}} g_{L}^{2} \sin \theta_{C}^{2} f_{D}^{2} M_{D}^{2} \frac{1}{M_{s}^{2}}(1+F(x, y)),
$$

where $M_{D}$ and $f_{D}$ are the $D^{0}$ mass and decay constant respectively; $M_{s}$ is the leptoquark mass; $x=\left(M_{\tilde{s}} / M_{s}\right)^{2}$ and $y=\left(m_{\tilde{e}} / M_{s}\right)^{2}$ with $M_{\tilde{s}}$ and $m_{\tilde{e}}$ being the sleptoquark and selectron masses and

$$
F(x, y)=\frac{x^{2}-y^{2}+2 x y \ln \frac{y}{x}}{(x-y)^{3}}
$$

parametrizes the sleptoquark-selectron loop contribution. We wish to translate (5.3) to a bound on the leptoquark parameters. To this end we must estimate $F(x, y)$. It is straightforward to show that:

$$
\frac{2}{3(x+y)} \leq F(x, y) \leq \frac{1}{x+y}
$$


Note that $(x+y)$ is likely to be $\sim 1$ since $\tilde{s}$ gets its mass from the supersymmetric parameter $M, \tilde{e}$ gets its mass from soft supersymmetry breaking terms while $s$ gets its mass from both sources. It is therefore reasonable to estimate that

$$
F(x, y) \sim 1
$$

Substituting this estimate in $\Delta M_{D}$ and using the experimental bound $\Delta M_{D}<1.5 \cdot 10^{-4} \mathrm{eV}$ at $95 \%$ CL we find the bound:

$$
\frac{M_{s}}{g^{2}} \geq 6.3 \mathrm{TeV}
$$

Comparing (5.7) to (5.2) we see that the FCNC bound on our $s$ leptoquark is somewhat stronger than the unavoidable FCNC bound. We wish however to stress that one is not likely to do better in any model which is natural and supersymmetric. This is due to two reasons:

(i) The minimal bound in (5.2) is achieved by fine tuned balance between FCNC processes in the down and up sector $[7,8]$. Since we are interested in presenting a model which is natural and not fine-tuned, we cannot have such a balance. At best, we can "clean" one of the two quark sectors of FCNC as we did: By using a QSA model we avoided the more severe FCNC of the down sector, and were left to deal only with FCNC in the up sector. (ii) In a supersymmetric model there is always an additional contribution to $D-\bar{D}$ mixing from the sparticle loop, and this necessarily strengthens the FCNC bound.

Another important remark on the bound (5.7) is that it is irrelevant for light leptoquarks: The $\pi$ decay bound (5.1) being linear in the coupling $g$ rather than quadratic, dominates when the leptoquark mass is below 1.9 TeV. Therefore, the bound on the leptoquark parameters is just the minimal unavoidable bound if $M_{s}<1.9 \mathrm{TeV}$.

Summarizing, our model avoids all the high scale bounds on the leptoquark parameters. For leptoquark masses below $1.9 \mathrm{TeV}$ the model does not impose any further bound beyond the absolutely unavoidable (5.1), while for heavier leptoquarks the dominant bound is (5.7). This latter bound is somewhat stronger than the unavoidable $(5.1,5.2)$, but we think it unlikely that it could be improved in any natural supersymmetric model.

\section{Summary and outlook}

We showed that the horizontal symmetries which are usually used to explain the pattern and hierarchy in the quark mass matrices can also be used to suppress unwanted leptoquark couplings. We presented a particular model of a first generation leptoquark 
with a horizontal symmetry and found that the unwanted couplings are naturally suppressed and the phenomenological constraints that may arise from the unwanted couplings are almost completely circumvented.

In concluding, we wish to point to a possible extension in the use of the horizontal symmetry protection mechanism. We showed that the horizontal symmetries can protect nonchiral couplings and nondiagonal couplings in the quark sector but they could actually be used to protect also the nondiagonal couplings in the lepton sector and it is possible that one may even progress one step further, drop altogether the conservation of all lepton and baryon numbers and provide sufficient protection to all the unwanted couplings via horizontal symmetries.

Acknowledgements: We thank Neil Marcus and Yossi Nir for useful remarks.

\section{References}

[1] M. Derrick et al. (ZEUS Collab.), Phys. Lett. 306B (1993) 173; I. Abt et al., (H1 Collab.) Nucl. Phys. B396 (1993) 3.

[2] See e.g. composite models: L.F. Abbott and E. Farhi, Phys. Lett. 101B (1981) 69; Nucl. Phys. B189 (1981) 547; H. Harari Phys. Lett. 86B (1979) 83; technicolour models: E. Farhi and L. Susskind, Phys. Rev. D20 (1979) 3404; superstring inspired models: J.L. Hewett and T.G. Rizzo, Phys. Rep. 183 (1989) 193.

[3] J.C. Pati and A. Salam, Phys. Rev. D10 (1974) 275.

[4] H. Georgi and S.L. Glashow Phys. Rev. Lett. 32 (1974) 438.

[5] O. Shanker, Nucl. Phys. B204 (1982) 375; B206 (1982) 253.

[6] W. Buchmüller and D. Wyler, Phys. Lett. 177B (1986) 377.

[7] M. Leurer, Phys. Rev. Lett. 71 (1993) 1324.

[8] M. Leurer, Phys. Rev. D49 (1994) 333.

[9] S. Davidson, D. Bailey, B.A. Campbell Zeit. fur Physik 61 (1994) 613.

[10] M. Leurer, Y. Nir and N. Seiberg, Nucl. Phys. B298 (1993) 319.

[11] Y. Nir and N. Seiberg, Phys. Lett. 309B (1993) 337. 
[12] M. Leurer, Y. Nir and N. Seiberg, Nucl. Phys. B420 (1994) 468.

[13] C.D. Froggatt and H.B. Nielsen, Nucl. Phys. B147 (1979) 277.

[14] F. Abe et al. (CDF Collab.), Fermilab preprint Fermilab-pub-94/097-E, submitted to Phys. Rev. D.

[15] The LEP Collaborations and the LEP electroweak working group, CERN preprint CERN/PPE/93-157. 